\title{
Prevention of early onset group B streptococcal infection: screen, treat, or observe?
}

For the past 15 years, North Americans have struggled to quantify and cost neonatal early onset group B streptococcal (EOGBS) infection, and to outline strategies for its prevention. A 24 page document has now been issued by the Centers for Disease Control in Atlanta, ${ }^{1}$ which has been endorsed by the American College of Obstetricians and Gynecologists, ${ }^{2}$ and by the American Academy of Pediatrics. $^{3}$

The core recommendation is the use of penicillin G, given intravenously to women in labour, to reduce the risk of neonatal (and maternal) EOGBS infection. The guidelines discuss the use of one of two strategies to decide which women should receive intrapartum antibiotic prophylaxis. One strategy is based on rectal and vaginal surveillance cultures taken at 35-37 weeks gestation, with intrapartum antibiotics given to all group B streptococcal carriers. The other strategy identifies women who are treated on the basis of risk factors for neonatal sepsis, but without prenatal screening cultures.

These recommendations revise pre-existing guidelines about which paediatricians and obstetricians could not agree, and which, consequently, were not widely implemented.

Over the same 15 year period in the United Kingdom, an eerie silence on the same subject has prevailed. The incidence of EOGBS infection in the UK is almost certainly lower than that in the USA, where it is estimated that in 1990, a national incidence of 1.8 cases per 1000 live births caused 7600 EOGBS neonatal infections and 310 neonatal deaths. ${ }^{1}$

\section{Epidemiology}

Group B streptococcus, or Streptococcus agalactiae, although known about for decades, only emerged as a major perinatal pathogen in the 1970 s. It is the predominant cause of early onset neonatal infection in North America, ${ }^{1}$ Australia, ${ }^{4}$ in almost all developed countries, and is an increasing problem in developing countries, as they become more industrialised. ${ }^{5}$

Group B streptococcus is a commensal of the gastrointestinal tract and vagina, and colonises $10 \%$ to $30 \%$ of pregnant women, both in the USA ${ }^{1}$ and the UK. ${ }^{6}$ The incidence of neonatal EOGBS infection is highest in babies born preterm and, in the USA, to mothers who are young ( $<20$ years old), black, and primiparous. ${ }^{7}$ Infection is acquired intrapartum, by the ascending route from the maternal perineum to the amniotic fluid bathing the fetal lungs where it causes pneumonia. Maternal risk factors increasing the risk of neonatal EOGBS infection are spontaneous preterm onset of labour ( $<37$ weeks gestation), prolonged rupture of membranes ( $>18$ hours), maternal fever $\left(>37.5^{\circ} \mathrm{C}\right)$ and a previously infected baby. ${ }^{178}$ In the USA the incidence of EOGBS neonatal infection is 1 to 4 per 1000 live births. It is comparable in white Australians, but even higher in Australian aboriginal babies. ${ }^{4}$

In the United Kingdom, despite the comparable level of maternal colonisation, the rate of EOGBS infection is often quoted as much lower than that in the USA, although there is scanty evidence for such statements. A study in 1978-9 found 66 cases in 226899 live births, an incidence of 0.3 cases per 1000 live births, ${ }^{9}$ but there have been no comparable studies published since then. A 10 year prospective study in Oxford, published in this issue (p F148-50), ${ }^{10}$ shows a constant rate of 0.5 cases per 1000 live births. The socioeconomic status of this population is unlikely to be nationally representative, and national data are urgently needed.

Based on the Oxford data alone,$^{10}$ and extrapolating from US mortality data, ${ }^{1}$ around 300 babies born in the UK each year develop EOGBS infection, of whom 12 will die.

\section{History}

Although others have studied group B streptococcus before, we owe much of the credit for our understanding of EOGBS infection to the pioneering work of Boyer and Gotoff in Chicago, USA. Their important epidemiological studies showed that about three quarters of women whose babies developed EOGBS infection had "risk factors" for sepsis as outlined above, but a quarter of the babies were full term babies with no obvious risk factors. ${ }^{8}$ Boyer and Gotoff set up a programme in which women were screened for carriage by vaginal culture at 26-28 weeks gestation. They performed a randomised controlled trial in which colonised women with risk factors were given intrapartum ampicillin or placebo, and proved that intrapartum antibiotics could reduce or even prevent neonatal group B streptococcal sepsis (and maternal complications), and reduce neonatal colonisation with the bacterium. ${ }^{11}$

This seminal study and others ${ }^{12}{ }^{12}$ were the basis for the 1992 guidelines issued by the American Academy of Pediatrics. ${ }^{14}$ Cynics might feel that development of the guidelines was assisted by pressure from the Group B Strep Association, a parent support group founded in 1990, who in mid 1992 called for universal screening, a call that was swiftly followed by a statement from the American Trial Lawyers Association.

However, the 1992 US guidelines could not prevent the $25 \%$ or so cases without risk factors, and cultures taken at 26-28 weeks identified as carriers women who subsequently became negative by delivery, while they failed to detect all new carriers. ${ }^{13}$ The policy of screening at 26-28 weeks and selective intrapartum chemoprophylaxis identified only about $60 \%$ of infected babies and was expensive. The obstetricians preferred not to screen but to treat women with "risk factors," which was cheaper and had the potential to prevent almost as many cases.

Other options were tried and results reported, although because of problems with study design, trials were longitudinal and observational rather than randomised and controlled. Jeffery and colleagues showed that screening at 26-28 weeks gestation and treating all colonised women with intrapartum antibiotics, regardless of risk factors (universal intrapartum chemoprophylaxis of carriers), led 
to a sustained reduction in EOGBS infection..$^{15}$ Siegel and colleagues gave a single dose of intramuscular penicillin to all neonates (universal neonatal chemoprophylaxis) which was almost as effective in preventing infection. ${ }^{16}$

Not all preventive tactics are beneficial. Some of the adverse effects, such as cost, are reasonably easy to quantify, others much harder. The risk of maternal anaphylaxis due to intravenous antibiotic use is often cited as being about 1 in 10000 women, ${ }^{1}$ and the risk of fatal anaphylaxis as about 1 in 100000 women. These are estimates, however, whose scientific basis, if any, has been lost in the mists of time and of circular cross referencing. Even if fatal maternal anaphylaxis is rare, it does occur, and babies may die or be damaged by non-fatal maternal anaphylaxis. ${ }^{17}$

\section{Cost-benefit analyses}

Gilbert and colleagues ${ }^{18}$ compared four strategies for prevention of EOGBS infection. These were:

- Screening cultures at 26-8 weeks; universal intrapartum chemoprophylaxis of all carriers

- Screening cultures at 26-8 weeks; selective intrapartum chemoprophylaxis of carriers with risk factors

- No screen; intrapartum chemoprophylaxis for women with risk factors

- Intrapartum chemoprophylaxis until 37 weeks gestation; screening cultures at 37 weeks; and universal intrapartum chemoprophylaxis for all carriers

This last strategy, which was subsequently recommended by the Centers for Disease Control for introduction in the USA, prevented most cases, but the third strategy was the cheapest. All strategies were "cost-effective," compared with using no prevention strategy, given that the incidence of group B streptococcal infection in Australia is relatively high. ${ }^{4}$

Mohle-Boetani et $a l^{19}$ compared the costs of three strategies, one based on screening cultures with selective prophylaxis (Gilbert's strategy 2), one based on epidemiological risk factors without screening (Gilbert's strategy 3), and one on a hypothetical vaccine. The vaccine fared best, but based on US epidemiological data, the other two options cost US $\$ 28800$ (screening cultures) and $\$$ US12 200 (risk factors) for each case prevented, and as many cases were prevented using epidemiological risk factors alone as by screening. These authors did not consider the costs of adverse events due to chemoprophylaxis. In their analysis Mohle-Boetani et al calculated how the costs of intervention varied with the incidence of EOGBS infection. The cost of introducing the risk factor strategy was greater than the calculated cost of the disease until the incidence of EOGBS infection exceeded 0.6 per 1000 live births. The cost of introducing the screening culture strategy was greater than the cost of disease until the incidence of infection exceeded 1.2 per 1000 live births.

If the US figures of cost of disease can be realistically extrapolated to other countries, this brings up a fifth option, one which is already being practised in many countries, including the United Kingdom, of masterly inactivity. The inactivity option is "economically justified" whenever the incidence of EOGBS infection is below 0.6 per 1000 live births, at least according to these figures, although putting an exact price on a life saved or handicap prevented is an inexact and uncomfortable procedure.

\section{Missing data}

Longitudinal studies of antibiotic chemoprophylaxis need to monitor carefully its use and the occurrence of adverse events. As these are rare it will be important to combine the experience of many centres and even many countries to document rates of anaphylaxis, and of maternal and neonatal mortality or morbidity resulting from anaphylaxis.

More precise national epidemiological data on the incidence, morbidity, and mortality of neonatal EOGBS infection are badly needed for the United Kingdom. Only on the basis of such data can a rational decision be made on whether the current lack of activity on prevention of neonatal GBS infection is justified, or whether prevention strategies should be considered.

Early onset neonatal group B streptococcal infection is an important public health problem in much of the world. Various preventive strategies of varying intensity and expense are available, depending on the local and national epidemiology and resources. When the incidence of EOGBS infection is low, say below 0.6 per 1000 live births, expensive preventive measures may not be justified.

Royal Alexandra Hospital for Children,

DAVID ISAACS

University of Sydney

Westmead,

NSW 2145

Australia

Email:davidi@nch.edu.au

1 Centers for Disease Control. Prevention of perinatal group B streptococcal disease: a public health perspective. Morbid Mortal Week Rep 1996; 45(RR7):1-24

2 American College of Obstetricians and Gynecologists. Prevention of earlyonset group B streptococcal disease in newborns. ACOG Comm Opin 1996;173:1-8

3 American Academy of Pediatrics. Revised guidelines for prevention of earlyonset group B streptococcal (GBS) infection. Pediatrics 1997;99:489-96.

4 Australasian Study Group for Neonatal Infections. Early-onset group B streptococcal infections in Aboriginal and non-Aboriginal infants. Med $\mathcal{F}$ Aust 1995;163:302-6.

5 Walsh JA, Hutchins S. Group B streptococcal disease: its importance in the developing world and prospect for prevention with vaccines. Pediatr Infect Dis f 1989;8:271-6.

6 Easmon CSF. The carrier state: group B streptococcus. F Antimicrob Chemother 1986;18 (Suppl A):59-65.

7 Schuchat A, Deaver-Robinson K, Plikaytis BD, et al. Multi state case-control study of maternal risk factors for neonatal group B streptococcal disease. Pediatr Infect Dis f 1994;13:623-9.

8 Boyer KM, Gadzala CA, Burd LI, Fisher DE, Paton JB, Gotoff SP. Selective intrapartum chemoprophylaxis of neonatal group B streptococcal early-onset disease. I. Epidemiologic rationale. F Infect Dis 1983;48:795801 .

9 Mayon-White RT. The incidence of GBS disease in neonates in different countries. Antibiot Chemother 1985;35:17-27.

10 Moses LM, Heath PT, Williamson AR, Jeffery HE, Isaacs D. Early onset group B streptococcal neonatal infection in Oxford 1985-96. Arh Dis Child 1998;79:F148-50.

11 Boyer KM, Gotoff SP. Prevention of early-onset neonatal group B streptococcal disease with selective intrapartum chemoprophylaxis. $N \mathrm{Engl} F \mathrm{Med}$ 1986;314:1665-9.

12 Yow MD, Mason EO, Leeds LJ, Thompson PK, Clark DJ, Gardner SE. Ampicillin prevents intrapartum transmission of group B streptococcus 7AMA 1979;241:1245-7.

13 Easmon CSF, Hastings MJG, Deeley J, Bloxham B, Rivers RPA, Marwood $\mathrm{R}$. The effect of intrapartum chemoprophylaxis on the vertical transmission of group B streptococci. Br F Obstet Gynaecol 1983;90:633-5.

14 Committee on Infectious Diseases and Committee of the Fetus and Newborn. Guidelines for prevention of group B streptococcal (GBS) infection by chemoprophylaxis. Pediatrics 1992;90:775-8.

15 Jeffery HE, Moses Lahra M. Eight-year outcome of universal screening and intrapartum antibiotics for maternal group B streptococcal carriers.
Pediatrics 1988;101:http://www.pediatrics.org/cgi/content/full/101/1/E2.

16 Siegel JD, Cushion NB. Prevention of early-onset group B streptococcal disease: another look at single-dose penicillin at birth. Obstet Gynecol 1996;87:692-8

17 Heim K, Alge A, Marth C. Anaphylactic reaction to ampicillin and severe complication in the fetus. Lancet 1991;337:859-60.

18 Gilbert GL, Isaacs D, Burgess MA, et al. Prevention of neonatal group B streptococcal sepsis: is routine antenatal screening appropriate? Aust NZ F Obstet Gynecol 1995;35:120-6.

19 Mohle-Boetani JC, Schuchat A, Plikaytis BD, Smith JD, Broome CV. Comparison of prevention strategies for neonatal group B streptococcal infection. A population-based economic analysis. JAMA 1993;270:1442-8. 